\title{
A Survey on Network Routing Protocols in Internet of Things (IOT)
}

\author{
Hanumat Prasad Alahari \\ Department of Computer Science \& \\ Engineering,Research Scholar, \\ AcharyaNagarjuna University, Guntur. India.
}

\author{
Suresh Babu Yalavarthi, PhD \\ Prof. in Computer Science, \\ Department of Computer Science, \\ J.K.C. College, Guntur, \\ Andhra Pradesh, India.
}

\begin{abstract}
Internet of Things (IoT) is a paradigm that has gained more popularity in recent years. At a conceptual level, IoT refers to the interconnectivity among our everyday devices such as personal computers, laptops, tablets, smart phones, PDAs, and other hand-held embedded devices. These devices now communicate smartly to each other. The goal of the Internet of Things is to enable things to be connected anytime, anyplace, with anything and anyone ideally using any path/network and any service. Internet of Things is a new revolution of the Internet. Objects make themselves recognizable and they obtain intelligence by making or enabling context related decisions thanks to the fact that they can communicate information about themselves and they can access information that has been aggregated by other things, or they can be components of complex services. This paper surveys some of the standard and non-standard protocols that are used for network routing in IoT applications. It should be noted that we have partitioned the network layer in two sub layers: routing layer which handles the transfer the packets from source to destination, and an encapsulation layer that forms the packets. Encapsulation mechanisms will be out of scope of this paper. Six network layer routing protocols of IoT were discussed in this paper.
\end{abstract}

\section{Keywords}

IoT, PDAs , Routing Layer

\section{INTRODUCTION}

The Internet of Things (IoT) is a paradigm that has gained more popularity in recent years. At a conceptual level, IoT refers to the interconnectivity

among our everyday devices such as personal computers, laptops, tablets, smart phones, PDAs, and other hand-held embedded devices[1,2] as shown in figure-1. These devices now communicate smartly to each other. Moreover, connected devices equipped with sensors and/or actuators perceive their surroundings, understand what is going on, and perform accordingly. These interconnected device networks can lead to a large number of intelligent and autonomous applications and services that can bring significant personal, professional, and economic benefits resulting in the emergence of more data centric businesses. IoT devices have to make their data accessible to interested parties, which can be web services, smart phone, cloud resource, etc. Hence, IoT can't be seen as individual systems, but as a critical, integrated infrastructure upon which many applications and services can run. Some applications will be personalized such as digitizing daily life activities, others will be citywide such as efficient, delay-free transportation, and others will be worldwide such as global delivery systems. The goal of the Internet of Things is to enable things to be connected anytime, anyplace, with anything and anyone ideally using any path/network and any service. Internet of Things is a new revolution of the Internet. Objects make themselves recognizable and they obtain intelligence by making or enabling context related decisions thanks to the fact that they can communicate information about themselves and they can access information that has been aggregated by other things, or they can be components of complex services. Six years ago, for the first time, the number of "things" connected to the Internet surpassed the number of people. Yet we are still at the beginning of this technology trend. Experts estimate that, as of this year, there will be 25 billion connected devices, and by 2020,50 billion. Some estimate that by 2020 , $90 \%$ of consumer cars will have an Internet connection, up from less than 15 percent in 2015 Three and one-half billion sensors already are in the marketplace, and some experts expect that number to increase to trillions within the next decade. All of these connected machines mean much more data will be generated: globally, by 2018, mobile data traffic will exceed fifteen Exabyte's - about 15 quintillion bytes - each month. By comparison, according to one estimate, an Exabyte of storage could contain 50,000 years' worth of DVD-quality video. Further, research in IoT relies on underlying technologies such as real-time computing, machine learning, security, privacy, signal processing, big data, and others. Consequently, the smart vision of the world involves much of computer science, computer engineering, and electrical engineering. Greater interactions among these communities will speed progress to design the smart devices, Smart phones, Smart cars, Smart homes, Smart cities and a smart world. This paper surveys some of the standard and nonstandard protocols that are used for network routing in IoT applications. It should be noted that we have partitioned the network layer in two sub layers: routing layer which handles the transfer the packets from source to destination, and an encapsulation layer that forms the packets. Encapsulation mechanisms will be out of scope of this paper. Six routing protocols in IoT were discussed in this paper .

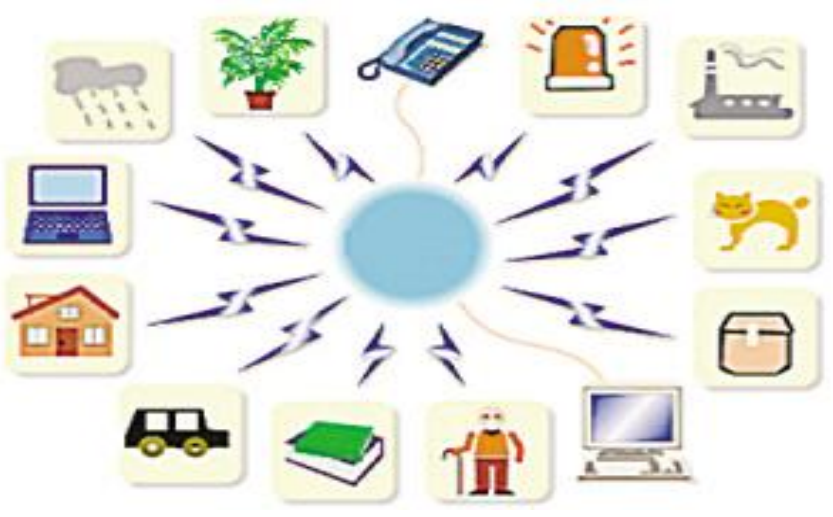

Figure-1 :Internet of Things 


\section{CHARACTERISTICS OF INTERNET OF THINGS(IOT)}

Some of the characteristics[3] of IOT that communicates with different varieties of physical devices. Device heterogeneity plays a very vital role in the technology of the IOT.

- Scalability : It must have the scalability as it is having many number of devices which are being over the vast network all the devices must be identified uniquely and the and the tags for the devices should be given properly.

- Information and knowledge management : when we consider IOT we don't need to give instructions every time to the machine the device is provided with the know ledge and information before it starts functioning and it takes decisions and find solutions on its own basing on the knowledge.

- Ubiquitous data exchange: In IOT where the devices are being connected through the internet and where the information is transferred. We have ubiquitous sensors where these are the intelligent sensors gather the information and transfer based on the given input.

- Optimized energy solution: We must be able to track even a low powered device and the consumer must be able to get the most optimal outcome.

- Localization and tracking capabilities: Must be able to track the devices and locate them with in a less duration.

- Self-Organization: It is needed to restore the services provided by the devices and to maintain the network connectivity.

\section{APPLICATIONS OF INTERNET OF THINGS (IOT)}

The world of IoT includes a wide variety of devices and diverse applications[8], which call for different deployment scenarios and requirements. Most of applications were used in our daily life

- Traffic Management: Where we don't require a traffic police to manage the traffic on roads and all the signal lights are automated where we don't require the use of the manual signals and where we can avoid the accidents.

- Driverless Cars: We don't require a driver in a car where all the and where is car is automatically controlled by the sensors of the car.

- Earthquake Detection: All the disasters like earthquake, tsunami etc. can be identified before it's going to happen through the IOT devices.

- Connected Medicine: Without going to the clinic the doctor can monitor the patients.

- Automotive Industry: Advanced Sensors are being equipped to all the cars, trains, buses etc. here we can use the RFID technology to increase the production and to satisfy the customer by providing more number of services.

- Independent Living: IOT application can be very useful for the aging people for reminding there medicines and activities on time and giving them support at the end of the lives.
- Retail And Marketing: Usage of the IOT in the marketing and the retail field is very beneficial. For example knowing the amount of stock present and for billing the amount through the usage of the RFID tags.

- Environment Monitoring: Usage of sensors and RFID where we can know more about the nature and where we can know the pollution caused to the nature.

- Transportation Industry: Where we can use them in the trains and buses for the collection of the fares and then we can avoid the toll gate system through the IOT.

- Agriculture And Breeding: Through the IOT we can use the high technology into the farming we can use the drone technology in to the agriculture and feeding the animals in the cattle.

\section{CHALLENGING ISSUES}

With advance of internet technology and development of social network, it is reasonable to expect that a new generation of Internet (also called future Internet) that will appear in the near future. In, several key technical issues [4] of IoT were pointed out. These challenges and open issues clarify that the dilemma of current Internet architecture requires great efforts to change.

- Security: Security providing might be difficult as the automation of the devices has been increased which created new security issues.

- Data management: As the communication between the devices is being done, every day between the devices lot of data is being generated and there is lot of information to be transferred from one place to another. Should check whether the exact data is being transferred or not. Data management plays a very important role in IOT.

- Storage management: As there is large amount of data generated. When the devices are being connected there would be a large amount of multimedia data which is being transferred they occupy a large amount of data and the other kind is random files where the it contains data regarding the devices these files doesn't occupy a huge amount of space but they are large in number they must be accessible very quickly whenever necessary.

- Server technologies: as the number of devices over the network area increases the request and the number of responses of the device also increases at the same time it totally depends on the server where we are running the interface. Response of the server to the request of the device should be done quickly. There should be no delay in the response to the client.

- Insecure authentication/authorization: We generally provide authentication to provide permission for the user to access the information and authorization is used to edit or change the data for that particular application and permission will be given by the administrator.

\section{NETWORK LAYER ROUTING PROTOCOLS}

This section discusses some of the standard and non-standard protocols[6] that are used for routing in IoT applications. It should be noted that we have partitioned the network layer in two sub layers: routing layer which handles the transfer the packets from source to destination, and an encapsulation layer that forms the packets. Encapsulation mechanisms will be out of scope of this paper. Six routing protocols in IoT were 
discussed in this section. RPL is the most commonly used one. It is a distance vector protocol designed by IETF in 2012 . CTP is a distance-vector routing algorithm that was developed as a solution to routing in WSNs. It stands as a predecessor to RPL and was considered the de-facto routing standard for Tiny OS. The Lightweight on-demand ad hoc distancevector routing protocol-next generation or LOADng is a lightweight variation of AODV for LLNs. It is designed based on the idea that LLNs are idle most of the time. Hence instead of adopting a proactive approach would generate unnecessary overhead, CORPL is a non-standard extension of RPL that is designed for cognitive networks and utilizes the opportunistic forwarding to forward packets at each hop. On the other hand, CARP and E-CARP is the only distributed hop based routing protocol that is designed for IoT sensor network applications. CARP and E-CARP is used for underwater communication mostly. Since it is not standardized and just proposed in literature, it is not yet used in other IoT applications.

RPL Protocol : RPL[7] is a distance-vector and a source routing protocol that is designed to operate on top of several link layer mechanisms including IEEE 802.15.4 PHY and MAC layers. These link layers could be constrained, potentially lossy, or typically utilized in conjunction with highly constrained host or router devices, such as but not limited to, low-power wireless or PLC (Power Line Communication) technologies. RPL mainly targets collectionbased networks, where nodes periodically send measurements to a collection point. A key feature of RPL is that it represents a specific routing solution for low power and lossy networks. The protocol was designed to be highly adaptive to network conditions and to provide alternate routes, whenever default routes are inaccessible. RPL provides a mechanism to disseminate information over the dynamically formed network topology. This mechanism uses Trickle to optimize the dissemination of control messages

Collection Tree Protocol (CTP) : In [5] CTP is a distancevector routing algorithm that was developed as a solution to routing in WSNs. It stands as a predecessor to RPL and was considered the de-facto routing standard for Tiny OS. It builds a tree-based topology with the root at the sink of the network, CTP uses adaptive beaconing mechanism to broadcast routing control messages. Moreover, CTP relied on a specific linklayer technology for topology formation, CTP was earlier known for its efficient energy consumption and high Packet Reception Ratio (PRR).
Lightweight on-demand ad hoc distance-vector routing protocol-next generation LOADng : The Lightweight ondemand ad hoc distance-vector routing protocol-next generation or LOADng [11] is a lightweight variation of AODV for LLNs. It is designed based on the idea that LLNs are idle most of the time. Hence instead of adopting a proactive approach would generate unnecessary overhead, LOADng follows a reactive approach in which routes are established towards destinations only when there is some data to send. LOADng is a reactive routing protocol, and found suitable for a more general traffic pattern. It does not have any node that performs special functions like the root and is hence not subjected to the subsequent problems that arise due to such a consideration. Also, due to its compressed and flexible data format, there is no possibility of fragmentation. It does not impose any strict source routing rules, hence it can accommodate applications which require a fixed MTU. However, LOADng might have a higher delay in the route discovery phase and might have higher control traffic overhead if the traffic flows are predominantly $\mathrm{P} 2 \mathrm{P}$.

CORPL Routing Protocol: In [12] CORPL will retain the Directed Acyclic Graph (DAG) based approach of RPL and at the same time introduce novel modifications to allow its application in Cognitive Radio environments. CORPL uses an opportunistic forwarding approach that consists of two key steps: selection of a forwarder set i.e., each node in the network selects multiple next hop neighbors, and a coordination scheme to ensure that only the best receiver of each packet forwards it (unique forwarder selection). In CORPL, each node maintains a forwarder set such that the forwarding node (next hop) is opportunistically selected. The DAG construction process in CORPL follows a similar procedure as in RPL. After detecting a vacant channel, the gateway node transmits a Destination Information Object (DIO) message. The forwarder set is constructed in such a way that the forwarding nodes are within the transmission range of each other. During the DIO transmission, each node also reports some additional information using the Option field of the DIO message. Each node updates the neighborhood information through the DIO message transmission. Based upon the neighborhood information, each node dynamically prioritizes its neighbors in order to construct the forwarder list.

Table-1: Study on various routing Protocols on Internet of Things

\begin{tabular}{|c|c|c|}
\hline Routing Protocol & Main Results & Simulation Used \\
\hline RPL & $\begin{array}{ll}\text { - } & \text { RPL showed better PRR and Energy consumption } \\
\text { - } & \text { RPL showed lesser churn } \\
\text { - } & \text { RPL showed high PRR } \\
\text { - } & \text { RPL had higher control-traffic overhead } \\
\text { - } & \text { RPL able to cater to variety of traffic patterns, } \\
\text { - } & \text { RPL is link-layer independent }\end{array}$ & $\begin{array}{c}\text { Contiki/ } \\
\text { Cooja }\end{array}$ \\
\hline CTP & $\begin{array}{l}\text { - In smaller networks, CTP showed better PRR. In larger } \\
\text { networks, } \\
\text { - } \quad \text { CTP showed high PRR } \\
\text { - } \quad \text { CTP is only collection-based }\end{array}$ & $\begin{array}{c}\text { Contiki/ } \\
\text { Cooja }\end{array}$ \\
\hline
\end{tabular}




\begin{tabular}{|c|c|c|}
\hline LOADng & $\begin{array}{ll}\text { - } & \text { LOADng caters to more general traffic pattern } \\
\text { - } & \text { LOADng has flexible and compressible } \\
\text { - } & \text { packet format } \\
\text { - } & \text { No single point of failure in LOADng } \\
\text { - } & \text { Longer route discovery phase in LOADng } \\
\text { - } & \text { More control traffic in LOADng if traffic is predominantly P2P }\end{array}$ & $\begin{array}{c}\text { Contiki/ } \\
\text { Cooja }\end{array}$ \\
\hline LOAD & $\begin{array}{ll}\text { - } & \text { In LOAD, control traffic / data traffic } \\
\text { - } & \text { LOAD routes longer than RPL routes } \\
\text { - } & \text { Higher delay in LOAD due to buffering during route-discovery } \\
\text { - } & \text { More collisions in LOAD due to flooding }\end{array}$ & NS2 \\
\hline CORPL & $\begin{array}{l}\text { - CORPL make use of Directed Acyclic Graph (DAG) like RPL } \\
\text { - } \quad \text { CORPL uses an opportunistic forwarding approach }\end{array}$ & $\begin{array}{l}\text { Contiki/ } \\
\text { Cooja }\end{array}$ \\
\hline CARP & $\begin{array}{l}\text { CARP is a multi-hop delivery of data to the sink for WSN. } \\
\text { CARP takes care of link quality while selecting the next-hop } \\
\text { node on a route to the sink }\end{array}$ & Real Time Test-bed \\
\hline E-CARP & $\begin{array}{l}\text { E-CARP is an enhancement upon CARP, } \\
\text { E-CARP is a location-free and greedy hop-by-hop routing } \\
\text { protocol for forwarding packets from sensor nodes to the sink } \\
\text { node in an energy efficient manner } \\
\text { - E-CARP does not differentiate the priority of different attributes }\end{array}$ & Test-bed \\
\hline
\end{tabular}

CARP Routing Protocol: In[9] Channel-aware Routing Protocol (CARP) is a multi-hop delivery of data to the sink for WSN. CARP obviates to the

Table-2: Comparison of Various protocols supports the Challenges Issues drawbacks such as link quality is explicitly taken into account for selecting the next-hop node on a route to the sink.

\begin{tabular}{|c|c|c|c|c|c|}
\hline \multicolumn{9}{|c|}{ Table-2: Comparison of Various protocols supports the Challenges Issues } \\
\hline Sno & Protocol Type & Server Technologies & Security & $\begin{array}{c}\text { Storage } \\
\text { Management }\end{array}$ & Data management \\
\hline 1. & RPL & Yes & NO & Yes \\
\hline 2. & CTP & Yes & NO & NO & NO \\
\hline 3. & LOADng & Yes & NO & Yes & Yes \\
\hline 4. & LOAD & Yes & NO & Yes & Yes \\
\hline 5. & CORPL & Yes & NO & NO & Yes \\
\hline 6. & CARP & NO & NO & Yes & \\
\hline
\end{tabular}

CARP quickly varying conditions of the underwater channel, the fact that two nodes can exchange short control packets correctly, may not be sufficient to guarantee that longer data packets are also going to be safely delivered". Generally, CARP is a location-free and greedy hop-by-hop routing protocol, whose performance is proved better than FBR[], and of its enhanced version EFlood. Link quality is explicitly considered when selecting a relay node for packet forwarding. The performance and applicability of CARP have been evaluated in the real ocean environment. However, there may have unnecessary control packets to be forwarded in CARP when selecting relay nodes for packet forwarding, and these control packets may be avoided in certain situations. Other characteristics that make CARP relay selection particularly suitable for implementing multi-hop routing in UWSNs include the following: (i) The use of simple topology information (hop count) for routing around connectivity holes and shadow zones, thus avoiding the well-known pitfalls of geographic routing; (ii) considering residual energy and buffer space, and (iii) taking advantage of power control, if available, for selecting 
transmission powers so that shorter control packets experience a similar Packet Error Rate (PER) of longer data packets.

E-CARP Routing Protocol: In [10] E-CARP, which is an enhancement upon CARP, to develop a location-free and greedy hop-by-hop routing protocol for forwarding packets from sensor nodes to the sink node in an energy efficient manner. Generally, CARP does not consider the reusability of sensory data collected previously by domain applications in the following time points, which induces sensory data packets forwarding which may not be beneficial to certain applications. Therefore, E-CARP allows the caching of sensory data at the sink node, for avoiding these data packets forwarding in the network. CARP requires to reply a PONG control packet whenever receiving a PING control packet, when selecting the most appropriate relay node for packet forwarding. This PING-PONG strategy may not be mandatory when the network topology is relatively steady. This observation drives us to improve the relay node selection strategy in CARP, and the relay node adopted previously is given a higher priority to be reused at this moment. Simulation results validate that our E-CARP can decrease the communication cost and increase the network capability to a large extent, especially when the ratio of packet size between control packets and sensory data packets is relatively large. ECARP does not differentiate the priority of different attributes. In fact, sensory data of attributes of more importance should be routed to SN with a higher priority. Besides, sensory data of a certain sensor node may vary following a spatial and/or temporal discipline.

\section{CONCLUSION}

This paper surveys some of the standard and non-standard protocols that are used for network routing in IoT applications. Six routing protocols in IoT were studied in this paper. RPL is the most commonly used

one. It is a distance vector protocol. CORPL is a non-standard extension of RPL that is designed for cognitive networks and utilizes the opportunistic forwarding to forward packets at each hop. On the other hand, CARP is the only distributed hop based routing protocol that is designed for IoT sensor network applications. CARP is used for underwater communication mostly. Since it is not standardized and just proposed in literature, it is not yet used in other IoT applications. E-CARP is an enhancement upon CARP, E-CARP is a location-free and greedy hop-by-hop routing protocol for forwarding packets from sensor nodes to the sink node in an energy efficient manner. ECARP does not differentiate the priority of different attributes. LOADng caters to more general traffic pattern LOADng has flexible and compressible packet format, No single point of failure in LOADng, Longer route discovery phase in LOADng, More control traffic in LOADng if traffic is predominantly P2P.

\section{REFERENCES}

[1] Gnawali, Omprakash, et al. "Collection tree protocol." Proceedings of the 7th ACM conference on embedded networked sensor systems. ACM, 2009.

[2] T. Winter, et al, "RPL: IPv6 Routing Protocol for LowPower and Lossy Networks," IETF RFC 6550, Mar. 2012

[3] IEEE 1905.1-2013, "IEEE Standard for a Convergent Digital Home Network for Heterogeneous Technologies," 93 pp., April 122013.

[4] A. Aijaz and A. Aghvami, "Cognitive machine-to-machine communications for internet-of-things: A protocol stack perspective," IEEE Internet of Things Journal, vol. 2, no. 2, pp. 103-112, April 2015.

[5] Al-Fuqaha, M. Guizani, M. Mohammadi, M. Aledhari, and M. Ayyash, "Internet of things: A survey on enabling technologies, protocols and applications," IEEE Communications Surveys Tutorials, vol. PP, no. 99, 2015.

[6] J. Granjal, E. Monteiro, and J. Sa Silva, "Security for the internet of things: A survey of existing protocols and open research issues," IEEE Communications Surveys Tutorials, vol. 17, no. 3, pp. 1294-1312, 2015,

[7] Salman, Tara. "Internet of Things Protocols and Standards.", 2015.

[8] Basagni, S., Petrioli, C., Petroccia, R., \& Spaccini, D. (2015). CARP: A Channel-aware routing protocol for underwater acoustic wireless networks. Ad Hoc Networks, 34, 92-104.

[9] Aijaz, Adnan, Hongjia Su, and Abdol-Hamid Aghvami. "CORPL: A routing protocol for cognitive radio enabled AMI networks." IEEE Transactions on Smart Grid 6.1 (2015): 477-485.

[10] Zhou, Z., Yao, B., Xing, R., Shu, L., \& Bu, S. (2015). ECARP: an energy efficient routing protocol for UWSNs in the internet of underwater things. IEEE Sensors Journal, 16(11), 4072-4082.

[11] . Verdiere, Axel, et al. "The Lightweight On-demand Ad hoc Distance-vector Routing Protocol-Next Generation (LOADng)." (2016). 